\title{
Pharmacist assessment of drug- gene interactions and drug-induced phenoconversion in major depressive disorder: a case report
}

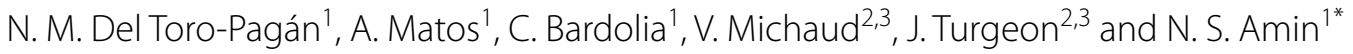

\begin{abstract}
Background: Response to antidepressant therapy is highly variable among individuals. Pharmacogenomic (PGX) testing presents an opportunity to guide drug selection while optimizing therapy outcomes and/or decreasing the risk for toxicity.

Case presentation: A patient with multiple comorbidities, including severe major depressive disorder (MDD), experienced adverse drug events and undesirable response to multiple antidepressant medications (i.e., bupropion, escitalopram, and venlafaxine). A clinical pharmacist assessed significant drug-gene, drug-drug, and drug-drug-gene interactions as well as other clinical factors to provide recommendations for antidepressant therapy optimization.
\end{abstract}

Conclusion: This case highlights the importance of PGx testing and the key role of pharmacists in identifying and mitigating drug-related problems and optimizing drug therapy in patients with MDD.

Keywords: Antidepressants, Case report, CYP2C19, CYP2D6, Pharmacogenetics, Pharmacogenomics

\section{Background}

Pharmacogenomics (PGx) is the study of human genome variants that impact drug response, typically through alterations in their pharmacokinetics or pharmacodynamics [1]. Overall, genetic differences are responsible for 15 to $30 \%$ of interpatient variability in drug disposition and response; however, for certain drugs, genetic differences can be responsible for up to $95 \%$ of the drug response variability [2]. When PGx results are known, drug-gene interactions (DGIs) and drug-drug-gene interactions (DDGIs) can be identified. DGIs are interactions involving a drug and coding variation in a gene leading to altered protein function or expression such as a

\footnotetext{
*Correspondence: namin@carekinesis.com

${ }^{1}$ Office of Translational Research and Residency Programs, Tabula Rasa

HealthCare, 228 Strawbridge Drive, Moorestown, NJ 08057, USA

Full list of author information is available at the end of the article
}

cytochrome P450 (CYP) isoenzyme (e.g., sertraline and CYP2C19 poor metabolizer phenotype [PM]), a receptor (e.g., morphine and $\mu$-opioid receptor) or transporter (e.g., simvastatin and SLCO1B1 decreased function). A DDGI results from the superimposition of a drug-drug interaction (DDI) on a DGI, often causing phenoconversion [3]. Phenoconversion is defined as the ability of intrinsic or extrinsic factors, such as DDIs, to modify a genotype-predicted phenotypic expression [4]. For example, an individual with a CYP2D6 ${ }^{*} 1 / * 1$ genotype predicted to exhibit a CYP2D6 normal metabolizer (NM) status is converted into a poor metabolizer (PM) while taking quinidine, a potent CYP2D6 inhibitor. Hence, the disposition of CYP2D6 substrates such as risperidone would be impaired in NM individuals during quinidine treatment, possibly increasing the risk of risperidonerelated toxicity. 
Genotyping can be done preemptively or reactively. Preemptive PGx testing allows for the availability of test results prior to the selection of a medication for which PGx clinical guidelines are available, thus enabling clinicians to better tailor drug selection [5]. Reactive PGx testing is generally completed under specific circumstances; for example, a patient experiencing pharmacotherapy failure or adverse drug events (ADEs) [6]. Organizations such as the Clinical Pharmacogenetics Implementation Consortium (CPIC) and the Dutch Pharmacogenetics Working Group (DPWG) facilitate the use of PGx in clinical practice and have developed clinical guidelines to ease drug and dose selection based on current evidence [7].

Recently, the therapeutic area of psychiatry has made efforts for achieving more precise treatments by considering interindividual differences in relevant genes for drug action [8]. Antidepressant response and efficacy vary among patients with major depressive disorder (MDD) [9]. Patients often experience long periods of poor management of depression symptoms and/or ADEs until the right medication and dose is established [10]. The initial antidepressant treatment response rate is about $50 \%$, while depression remission rate are around $37 \%$ [11]. Interpatient variability in antidepressant drug treatment response and efficacy can be attributed to environmental, physiological, and psychological factors, comorbidities, and genetic variability [12]. Evidence indicates that genes encoding for drug metabolizing enzymes that can impact pharmacokinetic parameters (e.g., CYP2C19, CYP2D6) may have a significant impact in the response and efficacy of such medications. In particular, CYP2C19 and CYP2D6 drug metabolizing enzymes are the primary focus for PGx in psychiatry because they predominantly contribute to the phase I metabolism of most currently available antidepressants, while the CYP2D6 enzyme contributes to the metabolism of many antipsychotics [13]. CYP2C19 and CYP2D6 genetic polymorphisms can affect the pharmacokinetic parameters (i.e., clearance, half-life, and plasma concentrations) of most antidepressants, thus increasing the risk for ADEs and impacting therapeutic outcomes [14]. Therefore, PGx testing can help predict antidepressant tolerability and response [15], potentially enabling a more safe, effective, and cost-effective treatment [16]. Although integrating PGx testing into the management of MDD has been implemented at some institutions, the widespread adoption of this practice has still to overcome many barriers such as, as insufficient evidence generation, data sharing and slow uptake of genomic information [17].

The case described herein demonstrates a unique approach leading to optimized antidepressant therapy in a patient. This approach consisted of a clinical pharmacist performing a PGx-informed assessment assisted by the proprietary clinical decision support system (CDSS), MedWise $^{\mathrm{TM}}$ which has been described elsewhere [18]. This CDSS incorporates PGx results in combination with the medication regimen to support the clinician on the identification of clinically significant DDIs, DGIs, DDGIs, and drug-induced phenoconversion. Other clinical factors are also considered to provide recommendations to optimize antidepressant therapy.

\section{Case presentation}

A 76-year-old female presented with MDD with inadequate response to antidepressants, post-traumatic stress disorder, obsessive compulsive disorder, generalized anxiety disorder, pseudobulbar affect, insomnia, restless leg syndrome, constipation, back pain, gastroesophageal reflux disease, hyperlipidemia, and glaucoma. Smoking status was not disclosed. Hepatic, kidney, and thyroid function laboratory values were all within normal limits (e.g., albumin, AST, ALT, creatinine, BUN, total protein, globulin, alkaline phosphatase, and bilirubin total, thyroid-stimulating hormone). The medications prescribed by the primary care provider (PCP) to manage her various medical conditions are listed in Table 1.

The patient's chief complaint was uncontrolled depression despite multiple attempts with various antidepressants. These medications include bupropion, escitalopram, and venlafaxine which all resulted in the patient experiencing ADEs and/or inadequate depression control. In brief and based on medical history, the patient was initially started on bupropion which was discontinued as she experienced uncontrolled shaking. Escitalopram was then used for 2 months and was discontinued due to an unknown reaction. Subsequently, venlafaxine was used for 3 months without favorable outcomes. This led to the introduction of other medications, such as: sertraline, risperidone and duloxetine. Additionally, this patient was also treated with quetiapine, although initiation date of treatment was unknown. The timeline for her antidepressant trials and other concomitant medications, including prescription and over the counter, is depicted in Table 2.

This participant had recently enrolled in the Program of All-inclusive Care for the Elderly (PACE). Within the PACE model, pharmacists and other healthcare practitioners collaborate to identify and mitigate medicationrelated problems [19]. PGx testing is one of the initiatives utilized to further improve the care of PACE participants. Being newly introduced to the management of this patient, a clinical pharmacist recommended a PGx test to optimize MDD management; this recommendation was accepted by the PCP. A DNA sample was collected via buccal swab and analyzed by a genetic laboratory 
Table 1 Patient's Medication List

\begin{tabular}{llll}
\hline Medical condition & Medication & Dose & Frequency \\
\hline MDD & Quetiapine IR & $100 \mathrm{mg}$ & Twice a day \\
PTSD & Quetiapine XR & $200 \mathrm{mg}$ & Daily \\
OCD & Risperidone & $0.5 \mathrm{mg}$ & Twice a day \\
GAD & Sertraline & $100 \mathrm{mg}$ & Daily \\
& Duloxetine DR & $30 \mathrm{mg}$ & Daily \\
Pseudobulbar effect & Dextromethorphan/quinidine & $20 / 10 \mathrm{mg}$ & Every 12h \\
Insomnia & Trazodone & $100 \mathrm{mg}$ & Daily at bedtime \\
Restless leg syndrome & Gabapentin & $100 \mathrm{mg}$ & Daily at bedtime \\
Constipation & Magnesium hydroxide suspension & $400 \mathrm{mg} / 5 \mathrm{~mL}$ & Daily at bedtime \\
Back pain & Polyethylene glycol 3350 & $17 \mathrm{~g}$ & Daily as needed \\
GERD & Acetaminophen & $500 \mathrm{mg}$ & Every 4h as needed \\
Hyperlipidemia & Pantoprazole DR & $40 \mathrm{mg}$ & Daily \\
Glaucoma & Famotidine & $20 \mathrm{mg}$ & Twice a day \\
\hline
\end{tabular}

Abbreviations: $D R$ Delayed Release, GAD Generalized Anxiety Disorder, GERD Gastroesophageal Reflux Disorder, IR Immediate Release, XR Extended Release, MDD Major Depression Disorder, OCD Obsessive Compulsive Disorder, PTSD Post-Traumatic Stress Disorder

(CQuentia, Memphis, TN; Genetic Response Report) and the clinical pharmacist was consulted to interpret relevant pharmacogenomic results. The patient was identified as a CYP2C19 IM, with a CYP2C19*2|*17 genotype, and as a CYP2D6 IM, with a CYP2D6*1|*4 genotype.

Although the clinical pharmacist assessed the complete drug regimen, only recommendations relevant to antidepressant and antipsychotic therapies will be discussed in this case report. It is also worth acknowledging that although the patient's chief complaint was uncontrolled depression and focus was on the MDD diagnosis, there are common symptoms with regards to her present psychiatric comorbidities. DGIs were considered relevant for the metabolism of duloxetine and risperidone (CYP2D6), and for the disposition of sertraline (CYP2C19). The CYP2D6 IM phenotype is associated with reduced enzyme activity and decreased clearance of CYP2D6 substrates. Hence, the risk of toxicity is increased for risperidone and to a lesser extent for duloxetine; it should be noted that the major metabolic pathway for duloxetine is through CYP1A2 (70\%), while the contribution of 2D6 is limited to $30 \%$. Similarly, a CYP2C19 IM has reduced enzyme activity, which results in decreased sertraline clearance and increased risk of toxicity.

The clinical pharmacist also identified three clinically significant DDIs (Table 3). Quinidine, a potent CYP2D6 inhibitor, is expected to inhibit the metabolism of risperidone and duloxetine. Such inhibition occurs regardless of the time of administration of the drugs as this interaction is mechanistically a non-competitive inhibition. The CDSS, which is based on algorithms and several pharmacological factors, was used to determine the presence of drug-induced phenoconversion (patent: WO 2019/089725). Quinidine inhibition of CYP2D6 resulted in phenoconversion whereby this patient's phenotype is converted from a CYP2D6 IM to a PM phenotype. When this interaction occurs, the plasma concentrations of risperidone and duloxetine are likely to be higher than predicted from the genotypic results alone. Lastly, DDIs were also identified by the CDSS on the CYP3A4 metabolic pathway; risperidone and quinidine are drugs with stronger affinity for the CYP3A4 enzyme than quetiapine. Consequently, these drugs are expected to competitively inhibit the metabolism of quetiapine when co-administered. When this interaction occurs, the plasma concentrations of quetiapine are likely to be significantly higher than predicted (CYP3A4 contributes to $75 \%$ of the total clearance of quetiapine), increasing the risk for toxicity. A similar mechanism of DDI occurs between sertraline - a CYP3A4 substrate with low affinity - risperidone, and quetiapine. CYP3A4 is responsible of $25 \%$ of the total clearance of sertraline, leading to moderately clinically significant changes in plasma concentrations of sertraline (if only this route of elimination is affected).

When performing the assessment and making recommendations, the clinical pharmacist considered several factors including previous unsuccessful medication trials, PGx test results and concomitant medications. Quetiapine has a low affinity for the dopamine 2 receptor, which is required for antipsychotic efficacy, therefore higher doses may be required for clinical effects for mood disorders and agitation. Additionally, quetiapine 
Table 2 PCP care - Reported antidepressant trials along with concomitant medications ${ }^{\dagger}$

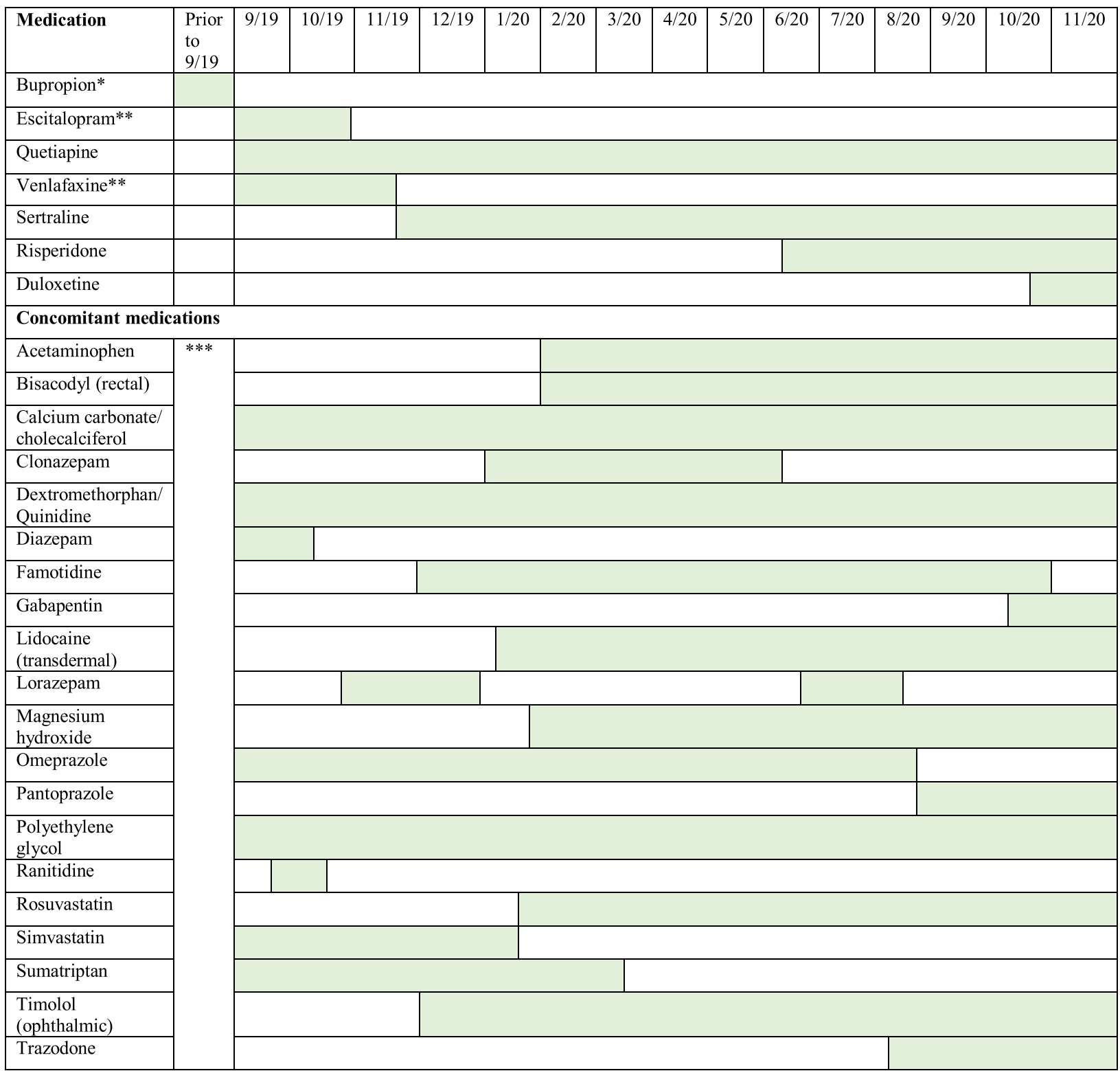

Abbreviations: PCP Primary Care Provider, ADE Adverse Drug Event

* Discontinued past medication trial because of ADEs

** Discontinued past medication trial for unknown reasons

*** Unknown status

${ }^{\dagger}$ Shaded area represents the period of time the patient was on each medication

has mixed results for the treatment of dementia psychosis and agitation [20]. Given this information and the DDI impacting the metabolism of quetiapine at CYP3A4, it was recommended that the PCP taper off the quetiapine while simultaneously optimizing the risperidone dose and frequency (targeting the lowest effective dose). Continued monitoring (e.g., EKG, palpitations) for risperidone was also suggested due to an increased risk of QTc prolongation caused by the presence of a DGI and a DDI at CYP2D6, and by the combination of quinidine [21], risperidone [22], rosuvastatin [23], quetiapine [24], and pantoprazole [25], which have all been associated with drug-induced QTc prolongation. Furthermore, it was recommended to optimize antidepressant therapy dosing based on clinical response and presence of ADEs. 
Table 3 Summary of Affinity and CYP450 Metabolic Pathways [18]

\begin{tabular}{|l|c|c|c|c|c|c|}
\hline \multicolumn{1}{|c|}{ Substance } & CYP1A2 & CYP2B6 & CYP2C9 & CYP2C19 & CYP2D6 & CYP3A4 \\
\hline $\begin{array}{l}\text { Dextromethorphan } \\
\text { Quinidine }\end{array}$ & & & & & $50 \%$ & $35 \%$ \\
\hline Duloxetine & $70 \%$ & & $15 \%$ & & & $30 \%$ \\
\hline Pantoprazole & & & & $90 \%$ & & \\
\hline Quetiapine & & & & & & $75 \%$ \\
\hline Risperidone & & & & & $60 \%$ & $40 \%$ \\
\hline Rosuvastatin & & & $10 \%$ & & & \\
\hline Sertraline & & $25 \%$ & & $40 \%$ & & $25 \%$ \\
\hline Trazodone & & & & & $25 \%$ & $55 \%$ \\
\hline
\end{tabular}

MedWise ${ }^{\mathrm{TM}}$ depicts the various degrees of binding affinity of a substrate for a specific enzyme using different colors (e.g., light yellow for weak affinity and dark yellow for moderate affinity. The percentages listed correspond to the use of the metabolic pathway for the substrate

\begin{tabular}{c|c|c|c|c|}
\hline Legend: & Meak Substrate & Moderate Substrate & Strong Substrate & Inhibitor \\
\hline
\end{tabular}

Over the next 8 weeks the aforementioned recommendations were accepted by the PCP, who before implementing had a thorough discussion with the patient's psychiatrist to account for other non-PGx factors. Tapering of the quetiapine dose was attempted and risperidone did not require a further increase in dose. Antidepressant therapy was optimized by increasing the duloxetine dose from $30 \mathrm{mg}$ to $60 \mathrm{mg}$ daily, the patient was monitored closely during dose escalation. It was determined that the dose of sertraline would be re-assessed based upon therapeutic response to these changes. The $\mathrm{PCP}$ reported that the patient was experiencing less anxiety and better control of depression as frequent monitoring was continued.

\section{Discussion and conclusions}

Uncontrolled depression is a major health issue that has significant individual and social-economic impact. In a systematic review conducted by Mrazek et al., it was shown that patients with uncontrolled depression have a $15 \%$ likelihood of suicide ideation compared to $6 \%$ for patients who respond appropriately to antidepressant therapy [26]. Hampton et al. estimated that each year over 25,000 patients in the United States present to the emergency department due to antidepressant-related ADEs [27]. Corponi et al. estimate that only around one third of patients with MDD, bipolar disorder, and/ or schizophrenia are compliant with their medications and are able to achieve full and stable remission [28]. Patients who do not have an adequate response to their antidepressant regimens are expected to have higher healthcare-related costs (about $\$ 10,000$ more yearly) than patients who do respond appropriately [26].

PGx test results combined with appropriate CDSS and clinical pharmacist interventions can help patients achieve depression remission quicker, decrease the number of ADEs, and potentially decrease healthcare costs related to trial-and-error approaches [29]. There are still conflicting results regarding the clinical utility and costeffectiveness of implementing PGx testing into routine clinical care despite PGx being a promising tool to guide treatment selection for patients with MDD [15]. This is explained in part by the fact that antidepressant plasma concentrations seem to poorly correlate with clinical efficacy [30]. Indeed, plasma concentrations remain an indirect estimation of drug concentrations in the effectcompartment (central nervous system), and several factors could explain this poor relationship such as distribution to the central nervous system limited by influx and efflux transporters expressed at the blood-brain barrier, inability to measure drug concentrations next to binding site on target receptors, and disease states [31]. However, dose adjustments based on PGx results for drug-metabolizing enzymes appear to provide more reliable information on the risk for ADEs [30]. Polymorphisms in the serotonin transporter and the tryptophan hydroxylase can also impact antidepressant response; however, insufficient data exists to support testing on these genes to modify antidepressant regimen [30, 32].

CPIC has developed evidence-based guidelines for sertraline therapy (Table S1) in patients who have readily available PGx information [33], while DPWG provides guidance for duloxetine [34], ,risperidone [35], and sertraline (Table S2) [36]. Around $40 \%$ of the total clearance of sertraline is mediated by CYP2C19 (Table 3), and its plasma concentrations are expected to be increased in CYP2C19 IMs. Additionally, a DDI was identified at CYP3A4 which could further increase the concentrations of sertraline (CYP2C19 DGI plus CYP3A4 DDI may 
decrease up to $65 \%$ of the total clearance of sertraline). Nevertheless, for IMs, both CPIC and DPWG guidelines recommend initiating sertraline therapy at the recommended starting dose [33, 36]. For the maintenance dose of sertraline, monitoring of ADEs is warranted and adjustments should be guided by therapeutic response (i.e., efficacy and toxicity), as mean steady-state plasma levels could increase with time. In this case, a close monitoring of potential side effects with sertraline was recommended due to the DGI combined with DDI. DPWG does not provide dosing recommendations for the duloxetine and CYP2D6 drug-gene pair since genetic variations have a limited effect on the plasma concentration of this medication (CYP2D6 mediated partial metabolic clearance is $30 \%$, while $70 \%$ is via CYP1A2) [34]. Due to the occurrence of phenoconversion at the CYP2D6 metabolic pathway, resulting in a CYP2D6 PM status, DPWG recommends reducing risperidone dose to $2 / 3$ of the standard dose to decrease the risk of central nervous system-related ADEs [35]. Additionally, this patient was concomitantly taking four QTc-prolonging medications: quinidine [21], risperidone [22], rosuvastatin [23], quetiapine [24], and pantoprazole [25]. DGIs and DDIs must be considered when evaluating QTc-prolongation risk. DGIs and DDIs can impact drug concentrations throughout the body, including intracellular concentrations in the heart - where the binding site to the rapid component of the delayed rectifier potassium channel ( $\mathrm{I}_{\mathrm{Kr}}$ or $\left.\mathrm{hERG}\right)$ is located - which can further increase the risk of QTc prolongation $[37,38]$. QTc prolongation is clinically relevant due to the risk of deterioration into Torsade de Pointes, ventricular fibrillation, and sudden death which can potentially be prevented with early recognition of risk factors $[37,39]$.

Lastly, PGx results can also help explain why this patient previously experienced undesirable effects with bupropion, escitalopram, and venlafaxine [32]. Bupropion is significantly cleared by CYP2B6 (50\%), for which we did not obtain genetic results. Bupropion is also a potent CYP2D6 inhibitor which likely impacted the metabolism of other concomitant medications at this time. This could have misled the causal clinical observations that side-effects were related to bupropion - not to concomitant treatments - leading to its discontinuation. CYP2C19 is responsible for $50 \%$ of escitalopram metabolic clearance while CYP2D6 contributes about $10 \%$. This patient is a CYP2C19 IM and phenotypically a CYP2D6 PM (due to phenoconversion). These conditions likely impaired the clearance of escitalopram, possibly leading to increased escitalopram plasma concentrations that could have explained the need for treatment discontinuation. Venlafaxine is metabolized by CYP2C19 (30\%) and CYP2D6 (50\%). Phenoconversion at CYP2D6 and the drug-gene interactions on both CYP450 isoforms likely caused increased venlafaxine concentrations (potential loss of $80 \%$ of the total clearance) which may have contributed towards undesirable ADEs.

A limitation of using PGx results to optimize antidepressant regimens is that not all health care professionals (e.g., physicians, pharmacists) are adequately trained to interpret these results. Furthermore, other factors such as, sex, smoking status, and comorbidities, can also contribute to interpatient variability of antidepressant response. In its current state, our unique approach does not consider other factors that can cause phenoconversion of the CYP450 enzymes, such as chronic inflammation [40]. We are studying closely this phenomenon which would be considered in future assessments [41]. Additionally, dietary habits that could affect drug response were not assessed.

CYP2C19 and CYP2D6 genetic variations and concomitant DDIs that lead to phenoconversion can significantly affect antidepressant tolerability and response. As illustrated by this patient case, some individuals are more susceptible to ADEs and may not respond appropriately to specific antidepressant therapy. Uncontrolled depression can result in noncompliance and potentially serious ADEs, such as suicidal ideation and behaviors. Implementing PGx testing into clinical practice with a clinical pharmacist that can identify and mitigate drug-related problems and optimize therapy can decrease ADEs, decrease polypharmacy, help patients achieve depression remission quicker, and potentially reduce healthcare-related costs. This case highlights the importance and clinical utility of PGx information and DGI data. Furthermore, it exemplifies pharmacist's role in helping other healthcare practitioners select the most precise antidepressant therapy, especially under conditions of genotype-to-phenotype mismatches due to concomitant drug administration.

\section{Abbreviations}

ADE: Adverse Drug Event; CPIC: Clinical Pharmacogenetics Implementation Consortium; CYP: Cytochrome P450; DDI: Drug-Drug Interaction; DDGl: DrugDrug-Gene Interaction; DGI: Drug-Gene Interaction; DPWG: Dutch Pharmacogenetics Working Group; DR: Delayed Release; GAD: Generalized Anxiety Disorder; GERD: Gastro-Esophageal Reflux Disease; IM: Intermediate Metabolizer; IR: Immediate Release; MDD: Major Depressive Disorder; NM: Normal Metabolizer; OCD: Obsessive Compulsive Disorder; PCP: Primary Care Provider; PGx: Pharmacogenomics; PM: Poor Metabolizer; PTSD: Post-Traumatic Stress Disorder; SSRI: Selective Serotonin Reuptake Inhibitor; XR: Extended Release.

\section{Supplementary Information}

The online version contains supplementary material available at https://doi. org/10.1186/s12888-021-03659-4.

Additional file 1: Table S1. CPIC Recommendations to Guide Sertraline Therapy Considering CYP2C19 Phenotype [33]. 
Additional file 2: Table S2. DPWG Recommendations to Guide Duloxetine and Risperidone Therapy Considering CYP2D6 Phenotype, and Sertraline Therapy Considering CYP2C19 Phenotype [34-36].

\section{Acknowledgements}

The authors would like to acknowledge Jasmine Dhanda, PharmD, BCGP for her support with the publication of this case report, and Dana Filippoli, MBE, MPH for her comprehensive review and comments pertaining to the contents of this manuscript.

\section{Authors' contributions}

NMDP, AM, CB, and NSA contributed to the proof outline. NMDP analyzed and interpreted the case. NMDP was the major contributor in writing the manuscript. AM, CB, NSA, VM, and JT substantially revised the work and provided conceptual ideas. All authors helped shape, read, and approved the final manuscript.

\section{Funding}

Not applicable.

\section{Availability of data and materials}

All data generated or analyzed during this study are included in this published article and its supplementary information files.

\section{Declarations}

\section{Ethics approval and consent to participate}

This case study was considered as exempt of review by the Biomedical Research Alliance of New York (BRANY) Internal Review Board (file \#21-15-105-427).

\section{Consent for publication}

Written informed consent was obtained from the patient for publication of this case report. A copy of the written consent is available for review by the Editor of this journal.

\section{Competing interests}

The authors declare that they have no competing interests.

\section{Author details}

'Office of Translational Research and Residency Programs, Tabula Rasa HealthCare, 228 Strawbridge Drive, Moorestown, NJ 08057, USA. ${ }^{2}$ Precision Pharmacotherapy Research \& Development Institute, Tabula Rasa HealthCare, Lake Nona, FL, USA. ${ }^{3}$ Faculty of Pharmacy, Université de Montréal, Montreal, Quebec, Canada.

Received: 25 June 2021 Accepted: 17 December 202

Published online: 20 January 2022

\section{References}

1. Relling MV, Evans WE. Pharmacogenomics in the clinic. Nature. 2015;526(7573):343-50.

2. Eichelbaum M, Ingelman-Sundberg M, Evans WE. Pharmacogenomics and individualized drug therapy. Annu Rev Med. 2006;57:119-37.

3. Bain KT, Matos A, Knowlton CH, McGain D. Genetic variants and interactions from a pharmacist-led pharmacogenomics service for PACE. Pharmacogenomics. 2019;20(10):709-18.

4. Tannenbaum C, Sheehan NL. Understanding and preventing drug-drug and drug-gene interactions. Expert Rev Clin Pharmacol. 2014:7(4):533-44.

5. Zhu Y, Moriarty JP, Swanson KM, et al. A model-based cost-effectiveness analysis of pharmacogenomic panel testing in cardiovascular disease management: preemptive, reactive, or none? Genet Med. 2021;23(3):461-70

6. Keeling NJ, Rosenthal MM, West-Strum D, Patel AS, Haidar CE, Hoffman JM. Preemptive pharmacogenetic testing: exploring the knowledge and perspectives of US payers. Genet Med. 2019;21(5):1224-32.
7. Volpi S, Bult CJ, Chisholm RL, Deverka PA, Ginsburg GS, Jacob HJ, et al. Research directions in the clinical implementation of pharmacogenomics: an overview of US programs and projects. Clin Pharmacol Ther. 2018;103(5):778-86.

8. van Schaik RHN, Müller DJ, Serretti A, Ingelman-Sundberg M. Pharmacogenetics in psychiatry: an update on clinical usability. Front Pharmacol. 2020;11:575540.

9. van der Weide J, Hinrichs JW. The influence of cytochrome P450 pharmacogenetics on disposition of common antidepressant and antipsychotic medications. Clin Biochem Rev. 2006;27(1):17-25.

10. Jha MK, Trivedi MH. Personalized antidepressant selection and pathway to novel treatments: clinical utility of targeting inflammation. Int J Mol Sci. 2018;19(1):233

11. Rush AJ, Trivedi MH, Wisniewski SR, Nierenberg AA, Stewart JW, Warden D, et al. Acute and longer-term outcomes in depressed outpatients requiring one or several treatment steps: a STAR* D report. Am J Psychiatry. 2006;163(11):1905-17.

12. van Westrhenen $R$, Aitchison KJ, Ingelman-Sundberg M, Jukić MM. Pharmacogenomics of antidepressant and antipsychotic treatment: how far have we got and where are we going? Front Psychiatry. 2020;11:94.

13. Tiwari AK, Souza RP, Müller DJ. Pharmacogenetics of anxiolytic drugs. J Neural Transm (Vienna). 2009;116(6):667-77.

14. Seeringer A, Kirchheiner J. Pharmacogenetics-guided dose modifications of antidepressants. Clin Lab Med. 2008;28(4):619-26.

15. Brown L, Vranjkovic O, Li J, Yu K, Al Habbab T, Johnson H, et al. The clinical utility of combinatorial pharmacogenomic testing for patients with depression: a meta-analysis. Pharmacogenomics. 2020;21(8):559-69.

16. Krebs K, Milani L. Translating pharmacogenomics into clinical decisions: do not let the perfect be the enemy of the good. Hum Genomics. 2019;13(1):39.

17. Dzau VJ, Ginsburg GS. Realizing the full potential of precision medicine in health and health care. JAMA. 2016;316(16):1659-60.

18. Bain $\mathrm{KT}$, Knowlton $\mathrm{CH}$, Turgeon J. Medication risk mitigation: coordinating and collaborating with health care systems, universities, and researchers to facilitate the design and execution of practice-based research. Clin Geriatr Med. 2017;33(2):257-81.

19. Bouwmeester C. The PACE program: home-based long-term care. Consult Pharm. 2012:27(1):24-30.

20. Blaszczyk AT, McGinnis KA, Michaels HN, Nguyen TN. Is it time to call it quits on low-dose quetiapine? Consult Pharm. 2015:30(5):287-90.

21. Tisdale JE. Drug-induced QT interval prolongation and torsades de pointes: role of the pharmacist in risk assessment, prevention and management. Can Pharm J (Ott). 2016;149(3):139-52.

22. Drolet B, Yang T, Daleau P, Roden DM, Turgeon J. Risperidone prolongs cardiac repolarization by blocking the rapid component of the delayed rectifier potassium current. J Cardiovasc Pharmacol. 2003;41 (6):934-7.

23. Plante I, Vigneault P, Drolet B, Turgeon J. Rosuvastatin blocks hERG current and prolongs cardiac repolarization. J Pharm Sci. 2012;101(2):868-78.

24. Hasnain M, Vieweg WV, Howland RH, Kogut C, Breden Crouse EL, Koneru $J N$, et al. Quetiapine, QTc interval prolongation, and torsade de pointes: a review of case reports. Ther Adv Psychopharmacol. 2014;4(3):130-8.

25. In brief: PPIs and Torsades de Pointes. Med Lett Drugs Ther. 2016:58(1509):153.

26. Mrazek DA, Hornberger JC, Altar CA, Degtiar I. A review of the clinical, economic, and societal burden of treatment-resistant depression: 19962013. Psychiatr Serv. 2014;65(8):977-87.

27. Hampton LM, Daubresse M, Chang HY, Alexander GC, Budnitz DS. Emergency department visits by adults for psychiatric medication adverse events. JAMA Psychiatry. 2014;71(9):1006-14.

28. Corponi F, Fabbri C, Serretti A. Pharmacogenetics in psychiatry. Adv Pharmacol. 2018;83:297-331.

29. Weinstein S, Carroll JC, Jukic S, Somma Mcgivney M, Klatt P. Perspec tives of a pharmacist-run pharmacogenomic service for depression in interdisciplinary family medicine practices. J Am Coll Clin Pharm. 2019;3(2):417-24

30. Kirchheiner J, Seeringer A, Viviani R. Pharmacogenetics in psychiatry--a useful clinical tool or wishful thinking for the future? Curr Pharm Des. 2010;16(2):136-44

31. Rizk ML, Zou L, Savic RM, Dooley KE. Importance of drug pharmacokinetics at the site of action. Clin Transl Sci. 2017;10(3):133-42. 
32. Kirchheiner J, Bertilsson L, Bruus H, Wolff A, Roots I, Bauer M. Individualized medicine - implementation of pharmacogenetic diagnostics in antidepressant drug treatment of major depressive disorders. Pharmacopsychiatry. 2003;36(Suppl 3):S235-43.

33. Hicks JK, Bishop JR, Sangkuhl K, Müller DJ, Ji Y, Leckband SG, et al. Clinical Pharmacogenetics implementation consortium (CPIC) guideline for CYP2D6 and CYP2C19 genotypes and dosing of selective serotonin reuptake inhibitors. Clin Pharmacol Ther. 2015;98(2):127-34.

34. PharmGKB. Annotation of DPWG Guideline for duloxetine and CYP2D6 2018 [updated November 2018. Available from: https://www.pharmgkb. org/guidelineAnnotation/PA166104942.

35. PharmGKB. Annotation of DPWG Guideline for risperidone and CYP2D6 2020 [updated May 2020. Available from: https://www.pharmgkb.org/ guidelineAnnotation/PA166104943.

36. PharmGKB. Annotation of DPWG Guideline for sertraline and CYP2C19 2018 [updated November 2018. Available from: https://www.pharmgkb. org/guidelineAnnotation/PA166104980.

37. Zeltser D, Justo D, Halkin A, Prokhorov V, Heller K, Viskin S. Torsade de pointes due to noncardiac drugs: most patients have easily identifiable risk factors. Medicine (Baltimore). 2003;82(4):282-90.

38. Morissette P, Hreiche R, Turgeon J. Drug-induced long QT syndrome and torsade de pointes. Can J Cardiol. 2005;21(10):857-64.

39. Banai S, Tzivoni D. Drug therapy for torsade de pointes. J Cardiovasc Electrophysiol. 1993;4(2):206-10.

40. Darakjian L, Deodhar M, Turgeon J, Michaud V. Chronic inflammatory status observed in patients with type 2 diabetes induces modulation of cytochrome P450 expression and activity. Int J Mol Sci. 2021;22(9):4967.

41. Gravel S, Chiasson JL, Turgeon J, Grangeon A, Michaud V. Modulation of CYP450 activities in patients with type 2 diabetes. Clin Pharmacol Ther. 2019;106(6):1280-9.

\section{Publisher's Note}

Springer Nature remains neutral with regard to jurisdictional claims in published maps and institutional affiliations.

- fast, convenient online submission

- thorough peer review by experienced researchers in your field

- rapid publication on acceptance

- support for research data, including large and complex data types

- gold Open Access which fosters wider collaboration and increased citations

- maximum visibility for your research: over $100 \mathrm{M}$ website views per year

At BMC, research is always in progress.

Learn more biomedcentral.com/submissions 